\title{
Global brain signal in awake rats
}

Yuncong Ma ${ }^{a}$, Zilu Ma ${ }^{a}$, Zhifeng Liang ${ }^{a, c}$, Thomas Neubergera,b, Nanyin Zhang ${ }^{a, b,{ }^{*}}$

a Department of Biomedical Engineering, The Pennsylvania State University, University Park,

PA, USA

b The Huck Institutes of the Life Sciences, The Pennsylvania State University, University Park,

PA, USA

${ }^{c}$ Current address: Institute of Neuroscience, Chinese Academy of Science, Shanghai, China

*Address for correspondence:

10 Nanyin Zhang, PhD

11 Professor of Biomedical Engineering and Electrical Engineering

12 Lloyd \& Dorothy Foehr Huck Chair in Brain Imaging

13 The Huck Institutes of the Life Sciences

14 The Pennsylvania State University

15 W-341 Millennium Science Complex, University Park, PA 16802, USA

16 Email: nuz2@psu.edu

\section{Acknowledgments}

The present study was partially supported by National Institute of Neurological Disorders and

Conflict of interest: none. 


\section{Compliance with Ethical Standards}

30 - Conflict of interest: none.

31 - Research involving Human Participants and/or Animals: The research involved animals. All procedures were conducted in accordance with approved protocols from the Institutional Animal Care and Use Committee (IACUC) of the Pennsylvania State University.

- Informed consent: N/A

- Funding: The present study was supported by National Institute of Neurological Disorders and Stroke (R01NS085200, PI: Nanyin Zhang, PhD) and National Institute of Mental Health (R01MH098003 and RF1MH114224, PI: Nanyin Zhang, PhD). 


\section{Abstract}

Although often used as a nuisance in resting-state functional magnetic resonance

44 imaging (rsfMRI), the global brain signal in humans and anesthetized animals has important

45 neural basis. However, our knowledge of the global signal in awake rodents is sparse. To bridge

46 this gap, we systematically analyzed rsfMRI data acquired with a conventional single-echo (SE)

47 echo planar imaging (EPI) sequence in awake rats. The spatial pattern of rsfMRI frames during

48 peaks of the global signal exhibited prominent co-activations in the thalamo-cortical and

49 hippocampo-cortical networks, as well as in the basal forebrain, hinting that these neural

50 networks might contribute to the global brain signal in awake rodents. To validate this concept,

51 we acquired rsfMRI data using a multi-echo (ME) EPI sequence and removed non-neural

52 components in the rsfMRI signal. Consistent co-activation patterns were obtained in extensively

53 de-noised ME-rsfMRI data, corroborating the finding from SE-rsfMRI data. Furthermore, during

54 rsfMRI experiments we simultaneously recorded neural spiking activities in the hippocampus

55 using GCaMP-based fiber photometry. The hippocampal calcium activity exhibited significant

correspondence with the global rsfMRI signal. These data collectively suggest that the global

57 rsfMRI signal contains significant neural components that involve coordinated activities in the 


\section{Introduction}

Compelling evidence suggests that the intrinsic brain activity plays an essential role in brain function. For instance, this spontaneously fluctuating brain activity consumes a major portion of brain's energy budget (termed as brain's dark energy (Raichle 2010)), much higher than that used for external tasks (Raichle 2006), and anomalies in intrinsic brain activity are tightly linked to brain disease (Zhang and Raichle 2010). The prominent utility of intrinsic brain activity, typically measured by resting state functional magnetic resonance imaging (rsfMRI), is to assess inter-areal resting-state functional connectivity (RSFC) (Biswal et al. 1995). Given its simplicity, whole-brain coverage and sensitivity, this method has been widely applied in human and animal studies and has revolutionized our understanding of brain network organization (Biswal et al. 2010; Smith et al. 2009; Liang et al. 2011).

An interesting research topic in intrinsic brain activity is the global brain signal, which is defined as the averaged rsfMRI signal across all brain voxels. The global signal was initially introduced as a nuisance to regress out in rsfMRI data preprocessing (Aguirre et al. 1997). The rationale underlying global signal regression is that distributed spontaneous neural activities are semi-random and out of phase, which will be cancelled out when rsfMRI signal is averaged across the whole brain. Therefore, the global rsfMRI signal can be treated as a nuisance dominated by non-neural fluctuations. Indeed, vascular signals from large veins show high temporal correlation to the global signal (Colenbier et al., 2019). As RSFC is assessed by temporal correlations between distributed rsfMRI signals, it is particularly susceptible to non-

81 neural confounds like head motion (Van Dijk et al. 2012; Power et al. 2012; Power et al. 2015;

82 Satterthwaite et al. 2012), respiration (Birn et al. 2008) and cardiac pulsation (Chang et al. 2009),

83 which all affect the rsfMRI signal globally and can cause systematic bias in RSFC quantification 
(Liu 2016). Therefore, regressing out the global rsfMRI signal represents an effective method for removing these non-neural artifacts (Ciric et al. 2018), and has been widely used (Fox et al. 2005). regression (Murphy et al. 2009) and it has been suggested that the global signal might have important neural components (Liu et al. 2018; Turchi et al. 2018; Scholvinck et al. 2010; Yang et al. 2014). Simultaneous recordings of electrophysiology and rsfMRI data revealed synchronized neural activity across the brain, which was also strongly correlated to brain-wide rsfMRI signals (Wen and Liu 2016; Scholvinck et al. 2010), showing that the global signal has neural basis. In addition, a series of studies reported that the global brain signal was tightly linked to the vigilance level in human subjects (Rack-Gomer and Liu 2012; Wong et al. 2012, 2013; Chang et al. 2016a; Liu et al. 2018), which suggests that the global signal plays an important functional role. Furthermore, altered global brain signal was reported in patients with 97 schizophrenia, indicating that the global signal could be an endophenotype of psychiatric disorders (Yang et al. 2017; Yang et al. 2014). Taken together, these studies have greatly advanced our understanding of the neural basis and physiologic function of the global signal in humans.

Recent studies in anesthetized animals have also shed light on potential neural 102 contributions to the global signal. Wide field imaging of calcium signals showed synchronized 103 neural activation across a large proportion of the cortex (Ma et al. 2016; Matsui et al. 2016). In 104 addition, brain's co-activation patterns in mice demonstrated specific phase relationship to the 105 global signal fluctuation (Gutierrez-Barragan et al., 2018). However, our knowledge of the global 106 signal in awake rodents is still lacking. Investigating this issue in awake animals is important as 
107 it avoids the potential confounding effects of anesthesia on animals' physiologic states and the 108 global signal (Gao et al. 2016; Liang et al. 2012b; Liang et al. 2015a; Ma et al. 2017; Smith et al.

109 2017; Hamilton et al. 2017), and permits linking brain activity measured to behavior (Liang et al.

110 2014; Dopfel et al. 2019). To bridge this gap, here we systematically investigate the global brain

111 signal using the awake rat fMRI approach established in our lab (Liang et al. 2011; Zhang et al.

112 2010; Perez et al. 2018; Dopfel and Zhang 2018). We first examine the spatial patterns of rsfMRI

113 volumes during peaks of the global signal. Our data demonstrate that the global signal in awake

114 rats might be linked to coordinated neural activities in the thalamo-cortical and hippocampo-

115 cortical networks. To confirm this concept, we acquire rsfMRI data using a multi-echo (ME) echo

116 planar imaging (EPI) sequence and use its advantage to remove non-neural components in

117 rsfMRI signal (Kundu et al. 2013; Kundu et al. 2012; Kundu et al. 2014). Consistent co-activation

118 patterns are obtained in extensively de-noised ME-rsfMRI data. Furthermore, during rsfMRI

119 experiments we simultaneously record spiking activities in the hippocampus using GCaMP-

120 based fiber photometry and find significant correspondence between hippocampal calcium

121 signal and the global rsfMRI signal. These data collectively suggest that the global signal in

122 awake rodents contains important neural components involving activities in the thalamo-cortical 123 and hippocampo-cortical networks.

\section{Methods and Materials}

\section{Animal Preparation}

128 Animal Care and Use Committee (IACUC) of the Pennsylvania State University. 92 adult male 129 Long-Evans rats (250-500g) were used in this study. 71 rats (133 scans) were scanned using 
130 single-echo rsfMRI (SE-rsfMRI), and 21 rats (43 scans) were scanned using ME-rsfMRI. Part of

131 the SE-rsfMRI data were used in previous publications (Ma et al. 2018; Ma and Zhang 2018)

132 and was reprocessed for the purpose of the present study. All animals were housed and 133 maintained on a $12 \mathrm{hr}$ light: $12 \mathrm{hr}$ dark schedule, and provided access to food and water ad libitum.

134 To minimize stress and motion during imaging at the awake state, animals were 135 acclimated to the scanning environment for 7 days (see details described in (Dopfel and Zhang 136 2018; Gao et al. 2016)). Briefly, rats were briefly anesthetized (5 min) under isoflurane (2-4\%) 137 and placed in a body and head restrainer. After this setup, isoflurane was discontinued, and 138 animals were allowed to regain full consciousness. The restrainer with the animal was then 139 placed in a mock MRI scanner where prerecorded sounds of MRI pulse sequences were played. 140 The exposure time started from 15 min on day 1, and was incrementally increased by 15 min 141 each day (days 2 and 3 ) up to 60 minutes (days 4, 5, 6 and 7). This setup mimicked the scanning 142 condition inside the magnet. A similar acclimation approach has also been used by other research groups (Bergmann et al. 2016; Yoshida et al. 2016; Chang et al. 2016b).

\section{MRI Experiments}

Using the method described in the acclimation procedure, rats were placed in an identical

147 head restrainer with a built-in birdcage radiofrequency coil. Isoflurane was discontinued after the 148 animal was set up. Imaging began $\sim 30$ min after rats were placed in the scanner while they were 149 fully awake. Image acquisition was performed at the High Field MRI Facility at the Pennsylvania 150 State University on a 7T Bruker 70/30 BioSpec running ParaVision 6.0.1 (Bruker, Billerica, MA). 151 After a localizer scan, T1-weighted structural images were acquired using a rapid imaging with 152 refocused echoes (RARE) sequence with the following parameters: repetition time $(T R)=$ 
$1531500 \mathrm{~ms}$, echo time $(\mathrm{TE})=8 \mathrm{~ms}$, matrix size $=256 \times 256$, field of view $(\mathrm{FOV})=3.2 \times 3.2 \mathrm{~cm}^{2}$,

154 slice number $=20$, slice thickness $=1 \mathrm{~mm}, \mathrm{RARE}$ factor $=8$, and repetition number $=8$. For the

155 SE-rsfMRI experiment, one to three SE-rsfMRI scans were acquired using a single-shot 156 gradient-echo EPI pulse sequence with the following parameters: TR = 1000ms, TE =15ms, flip

157 angle $=60^{\circ}$, matrix size $=64 \times 64, \mathrm{FOV}=3.2 \times 3.2 \mathrm{~cm}^{2}\left(\right.$ in-plane resolution $\left.=0.5 \times 0.5 \mathrm{~mm}^{2}\right)$,

158 slice number $=20$, slice thickness $=1 \mathrm{~mm} .600$ SE-rsfMRI volumes $(10 \mathrm{~min})$ were acquired for 159 each SE-rsfMRI scan.

21 animals (body weight $=307 \pm 19 \mathrm{~g}$ ) were scanned in an ME-rsfMRI experiment. The

161 setup was the same as the SE-rsfMRI experiment. As the scanner noise was louder for the ME-

162 EPI sequence, earplugs were used in rats to reduce acoustic noise during ME-rsfMRI data

163 acquisition. For each animal, one to three ME-rsfMRI scans were acquired using a single-shot

164 ME-EPI sequence (Fig. 1A) with TR = $1500 \mathrm{~ms}$, TEs = 7.6, 16.4, 25.2 and $34.0 \mathrm{~ms}$, flip angle = $16575^{\circ}$, matrix size $=48 \times 32, \mathrm{FOV}=2.4 \times 1.6 \mathrm{~cm}^{2}\left(\right.$ in-plane resolution $\left.=0.5 \times 0.5 \mathrm{~mm}^{2}\right)$, slice 166 number $=18$, slice thickness $=1 \mathrm{~mm} .400 \mathrm{ME}-\mathrm{rsfMRI}$ volumes $(10 \mathrm{~min})$ were acquired for each 167 ME-EPI scan.

\section{Data Analysis}

Analyses of SE-rsfMRI and ME-rsfMRI data were carried out using MATLAB 2017b (The

171 MathWorks, Inc., Natick, MA). Mathematica 11.3 (Wolfram Research, Inc., Champaign, IL), 172 MATLAB and ITK-SNAP (Yushkevich et al. 2006) were used for data visualization. 
176 publications (Ma et al. 2018; Liang et al. 2012a; Liang et al. 2013; Liang et al. 2015b; Liu and

177 Zhang 2019). First, head motion was estimated by frame-wise displacement (FD) in each rsfMRI

178 scan (Power et al. 2012). Volumes with large FD (>0.2mm) and their immediate preceding and

179 following volumes were removed. The first 10 rsfMRI volumes were also removed to ensure

180 steady-state magnetization. SE-rsfMRI scans with $>10 \%$ volumes scrubbed were excluded from

181 further analysis. Subsequently, the procedures of co-registration, spatial smoothing (Gaussian

182 kernel, FWHM $=0.75 \mathrm{~mm}$ ), motion-correction, nuisance regression of 6 motion parameters (3

183 translational and 3 rotational parameters) and signals from the white matter and ventricles were 184 respectively applied.

\section{ME-rsfMRI data preprocessing}

ME-rsfMRI data were used to separate neural and non-neural signals in rsfMRI data

(Kundu et al. 2013; Kundu et al. 2012; Kundu et al. 2014). This capacity is based on the premise

that neural activity-induced blood-oxygenation-level dependent (BOLD) signal change is TE

dependent, as BOLD changes originate from alterations in paramagnetic deoxy-hemoglobin

191 concentrations, which lead to changes in $\mathrm{T}^{*}{ }_{2}$ values. An innovative feature of the ME-EPI method

192 is that each rsfMRI volume was acquired at multiple TEs, which allowed us to examine voxel193 wise TE dependency of rsfMRI signal (see below).

194 Preprocessing of ME-rsfMRI data was similar to SE-rsfMRI data. The same motion 195 scrubbing method described above was used to remove volumes with FD $>0.2 \mathrm{~mm}$. The first 19610 volumes were also removed to ensure steady-state magnetization. ME-rsfMRI scans with > $19710 \%$ volumes scrubbed were excluded from further analysis. Motion parameters were then 198 estimated using moderately smoothed EPI images (FWHM=0.75mm) acquired at the first echo. 
199 Subsequently, we combined estimated matrices of motion parameters and the affine matrix from 200 co-registration, and applied the combined matrix to each volume in EPI. After that, images were 201 spatially smoothed (Gaussian kernel, FWHM $=0.75 \mathrm{~mm}$ ). The time course of each voxel was 202 detrended, and 6 motion parameters were regressed out.

203 To separate neural and non-neural signals, we employed a similar method established 204 by Kundu et. al. (Kundu et al. 2013; Kundu et al. 2012; Kundu et al. 2014), including the steps 205 of optimally combining ME data, spatial group ICA, dual-regression back reconstruction, and 206 computation of BOLD and non-BOLD weighting for each ICA component.

For each voxel in ME-rsfMRI images, Eq. 1 describes the MR signal as a function of TE, where $S(T E)$ is the signal amplitude at $T E, S_{0}$ is the signal amplitude when $T E$ is zero, and $T 2^{*}$ 209 is the time constant for T2* relaxation. For each scan, the rsfMRI signal of each brain voxel was 210 averaged across volumes for each $T E$, and $T 2 *$ was estimated by fitting signals at four TES to 211 Eq. 1. Then, signals acquired at four TEs were weighted averaged with the weight quantified 212 using Eq. 2, where $W_{T E_{i}}$ is the weight for the $i$-th $T E\left(T E_{i}\right)$. This step maximized the contrast-to213 noise ratio of rsfMRI data for group-ICA.

217 generate group-level independent components using GIFT v3.0b (component number = 100,

218 Fig. 1C) (Calhoun et al. 2001). Spatial maps and the corresponding time courses of all ICA 219 components were then generated for each individual scan using dual regression back 220 reconstruction (Calhoun et al. 2001). 
To differentiate neural and non-neural components in ME-rsfMRI data, BOLD (к) and non-

BOLD $(\rho)$ weights of each ICA component were decided. $\kappa$ and $\rho$ were pseudo- $F$-statistics that

measured how likely the BOLD signal changes were due to the change of $T_{2}^{*}$ (BOLD) or $S_{0}$ (non-

BOLD) in Eq. [1], as BOLD signal changes were $T 2^{*}$ dependent, whereas non-BOLD changes

were not. Specifically, the first order approximation of Eq. [1] can be further separated into two linear sub-models:

where $\triangle S(T E) / S(T E)$ is the signal change at a given TE. Signal changes of each voxel at different

TEs were fit to the two sub-models, and goodness-of-fit $(F)$ for Eq. 3 and 4 was calculated (i.e.

$F_{R_{2}^{*}}$ and $\left.F_{S_{0}}\right)$, respectively. The $\kappa$ and $\rho$ weights for each ICA component were obtained by

weighted averaging $F_{R_{2}^{*}}$ or $F_{S_{0}}$ of all voxels of a component using the voxel's ICA component

weight (i.e. $z$ value) as the weighting. BOLD components had high $\kappa$, whereas non-BOLD

components had high $\rho$. Fig. 1B shows the $\rho$ and $\kappa$ values for all ICA components, with $\rho$ being

sorted from the smallest to the largest. The elbow point of the sorted curve was noted as $\rho_{\text {elbow }}$

237 BOLD components (79 in total, referred to as neural components hereafter), and all other

238 components were recognized as non-BOLD components (21, Fig. 1C, referred to as non-neural

239 components hereafter). Notably, these criteria were highly stringent for defining neural

240 components. Dual regression back reconstruction was performed to derive the time courses of

241 ICA components for each individual scan. Lastly, time courses of all non-BOLD components and

242 the white matter signal were regressed out from optimally combined ME-rsfMRI data for each 
243 scan.

\section{4}

\section{5}

265 in the global signal.

\section{Generating global signal co-activation patterns}

For each rsfMRI scan, the time course of each voxel was first normalized to its mean, and the global signal was calculated as the average of normalized time courses across all brain voxels, and then temporally filtered to $0.01-0.1 \mathrm{~Hz} .15 \% \mathrm{rsfMRI}$ volumes with the highest global signal amplitude were selected for each scan, pooled together across all scans, and averaged to generate the global signal co-activation pattern (CAP). In addition, k-means clustering was applied to group these selected rsfMRI volumes based on their spatial similarity, and the mean CAP for each cluster was calculated. The same processing procedures were separately applied to SE-rsfMRI and ME-rsfMRI data.

\section{Extracting spatiotemporal patterns of the global signal}

To further investigate the spatiotemporal dynamics of the global signal, we extended our analysis to rsfMRI epochs surrounding local global signal peaks. Each global signal epoch was defined as rsfMRI volumes ( $9 \mathrm{~s}$ in duration) centered at a local global signal peak. Global signal epochs overlapping with each other were excluded from the analysis to ensure data independency. The volumes used for global epochs accounted for $55.8 \%$ of the total number of volumes in ME-rsfMRI data, and explained $75.0 \%$ of variance in the global signal. All global signal epochs were averaged to generate the mean spatiotemporal patterns of the global signal. This procedure was separately applied to SE-rsfMRI and ME-rsfMRI data. All global signal epochs were subjected to spatial group ICA to further extract individual brain networks involved 


\section{Surgery for calcium signal recording}

rsfMRI and calcium signal signals were simultaneously recorded $(n=4)$ using the method previously reported (Liang et al. 2017). Before imaging, stereotaxic surgery was conducted for virus injection and optic fiber implantation. Rats (350 - $450 \mathrm{~g})$ were anesthetized by intramuscular (IM) injections of ketamine $(40 \mathrm{mg} / \mathrm{kg}$ ) and xylazine $(12 \mathrm{mg} / \mathrm{kg})$. Buprenorphine $(1.0 \mathrm{mg} / \mathrm{kg})$ was injected subcutaneously (SC) as long-term post-surgery analgesia. Dexamethasone $(0.5 \mathrm{mg} / \mathrm{kg})$ was injected SC to prevent tissue inflammation. Rats were intubated with endotracheal catheter with a fiber-optic guide (Rivera et al. 2005), placed on a stereotaxic frame (David Kopf Instruments, Tujunga, CA), and ventilated with oxygen. The animal's heart rate and $\mathrm{SpO} 2$ were monitored and the body temperature was maintained at $37^{\circ} \mathrm{C}$ during surgery. A small craniotomy was made unilaterally over the dorsal hippocampus (dentate gyrus, $3.5 \mathrm{~mm}$ rostral and $2 \mathrm{~mm}$ lateral to bregma). AAV5.Syn.GCaMP6s (800-1000 $\mathrm{nl}$, Penn Vector Core) was injected through a micropipette syringe fitted with a glass pipette tip (Hamilton Company, Reno, NV) at three depths: $-2.6 \mathrm{~mm},-2.8 \mathrm{~mm}$, and $-3.0 \mathrm{~mm}(\sim 300 \mathrm{nl}$ at each depth). After virus injection, a fiber optic $(400 \mu \mathrm{m}$ core, $0.48 \mathrm{NA}, 2.5 \mathrm{~mm}$ ceramic ferrule, Thorlabs, Newton, NJ) was advanced to the injection site (depth: $-2.6 \mathrm{~mm})$. Five screws $(0.06$ inch in diameter, 0.125 inch in length, brass; McMaster-Carr, Aurora, $\mathrm{OH}$ ) were implanted along the temporal ridge of the rat skull. Dental adhesive and dental cement (ParaBond, COLTENE, Cuyahoga Falls, $\mathrm{OH}$ ) were applied to cover the skull and fix the implanted fiber. Rats were returned to home cages, and allowed for recovery and GCaMP expression for at least 4 weeks.

\section{Simultaneous calcium-rsfMRI signal recording}

A two-wavelength GCaMP fiber photometry system (Doric Lenses Inc., Quebec, Canada) 
290 was utilized for calcium signal recording (Fig. 2A) (Kim et al. 2016). GCaMP and Ca ${ }^{2+}$

291 independent fluorescent signals were alternatingly excited by a $465 \mathrm{~nm}(7 \mu \mathrm{W})$ LED and an

292 isosbestic wavelength $(405 \mathrm{~nm}, 0.75 \mu \mathrm{W})$ LED, respectively, both modulated at $400 \mathrm{~Hz}(50 \%$ duty

293 cycle for each wavelength). Power for the two LEDs was adjusted to achieve comparable 294 intensity of emitted fluorescent light for the two channels due to the different absorption rate of 295 the two wavelengths in the brain tissue and different light efficiency in the optic setup. Both 296 excitation sources were combined via a dichroic mirror in a mini-cube and coupled into a mono 297 fiber optic patch cable (400 nm core, 0.48NA, 7m long, Doric Lenses Inc., Quebec, Canada) 298 connected with the implanted optical fiber. Emitted fluorescent light was collected through the 299 same patch cable, separated by another dichroic mirror in the mini-cube, coupled via a fiber 300 launch (Thorlabs, Newton, NJ) and a 40×0.65 NA microscope objective (Olympus, Center Valley, 301 PA), and then focused into a photomultiplier (MiniSM 30035, SensL Technologies, Somerville, 302 MA). The converted signal was amplified (bandpass filtered at 0.3-1kHz, Dagan Corp., 303 Minneapolis, MN) and recorded using an NI-DAQ board (10kHz sampling rate, NI USB-6211, 304 National Instruments, Austin, TX) and custom-written LabVIEW code. TTL signals used to 305 control the two LED modules were also recorded. We used a time-division multiplexing strategy 306 to time-sequentially sample $465 \mathrm{~nm}$ and $405 \mathrm{~nm}$ excited fluorescent signals (Fig. 2). $405 \mathrm{~nm}$ 307 signals were regressed out from $465 \mathrm{~nm}$ signals to correct for fluorescence changes unrelated 308 to neuronal activity (Kim et al. 2016).

Along with GCaMP signal, SE-rsfMRI signal was simultaneously collected using the same 310 imaging parameters mentioned above. We acquired 1-3 scans in each session. For each animal, 311 we performed multiple sessions on separate days, which provided 20 scans in total.

\section{Results}


In this study we systematically investigated the global brain signal in awake rats. We first examined the spatial patterns of rsfMRI volumes at global signal peaks. The neural components

316 in the global signal spatial pattern were further investigated using ME-rsfMRI data and

317 simultaneously recorded calcium signal.

Co-activation patterns of the global rsfMRI signal

Fig. 3 shows the mean CAP generated by averaging 15\% rsfMRI volumes with the highest

321 global signal amplitude. This global signal CAP displayed well-organized network activities mainly involving the thalamocortical and hippocampocortical networks. In particular, regions in these two networks including the medial prefrontal, insular, anterior cingulate, retrosplenial and sensorimotor cortices, as well as medial dorsal thalamus, hippocampus and basal forebrain showed strong BOLD signal during global signal peaks. This result suggests that the global signal in awake rats might be attributed to coordinated activities in the thalamo-cortical and hippocampo-cortical networks. signal. To examine this possibility, k-means clustering $(\mathrm{k}=12)$ was used to group rsfMRI volumes based on their spatial similarity (Fig. 4). Fig. 4A displayed clusters of CAPs resembling

331 the mean global signal CAP (spatial correlation coefficient $=0.72 \pm 0.10$ ), which accounted for $33279 \%$ of total rsfMRI volumes selected. In contrast, several other cluster CAPs exhibited spatial 333 patterns quite different from the mean global signal CAP (Fig. 4B). These patterns occurred in a 334 minor portion (21\%) of rsfMRI volumes selected. None of them showed any patterns consistent 335 with known anatomical or network structures, and therefore, might be attributed to non-neural 
337 and non-neural contributions to the global signal, even after routine signal preprocessing.

Dissecting neural components in the global rsfMRl signal

ME-rsfMRI was employed to further differentiate neural and non-neural components in

341 the global rsfMRI signal. Fig. 1C shows both neural and non-neural components generated using

342 ME-rsfMRI data. rsfMRI signals from all non-neural components were regressed out from ME-

343 rsfMRI data, generating a de-noised rsfMRI dataset. The mean CAP of all global signal peaks

344 was then calculated using the same method described above. Our data showed that the mean

345 global signal pattern in de-noised ME-rsfMRI data (Fig. 5) was highly consistent with the mean

346 global signal CAP obtained from SE-rsfMRI data (Fig. 3C), including activated regions in the

347 thalamo-cortical and hippocampo-cortical networks, as well as the basal forebrain. Consistent

348 results between SE- and ME-rsfMRI data further validated that the global signal in awake rats

349 was linked to coordinated neural activities in these networks.

Spatiotemporal dynamics of the global signal

To examine the spatiotemporal dynamics of the global signal, we analyzed rsfMRI epochs surrounding local peaks of the global signal (9 s per epoch, Fig. 6A). Figs. 6B \& 6C show the

spatiotemporal patterns averaged across all global signal epochs for SE-rsfMRI and ME-rsfMRI data, respectively. As expected, the central volumes in both SE and ME-rsfMRI data were highly consistent with the mean global signal CAPs shown in Figs. $3 \mathrm{C}$ and 5, respectively, with strong activity in the thalamo-cortical and hippocampo-cortical networks. The involvement of these two to all global signal epochs in the ME-rsfMRI dataset, which provided two prominent ICA 
360 components representing the thalamo-cortical network and hippocampo-cortical network, 361 respectively (Fig. 7).

Fig. 6D shows frame-by-frame global signal amplitude averaged across all global signal epochs for SE- and ME-rsfMRI data, respectively. Results from both datasets displayed almost 365 identical global signal temporal profiles, which started $\sim 4$ s before the peak, sustained for $\sim 2-3$ $\mathrm{s}$ and then returned to baseline, with a total period of $\sim 8 \mathrm{~s}$.

Correspondence between the global rsfMRI signal and neuronal spikes in the hippocampus

To further validate that the global signal involved neural activity in the hippocampo-cortical network, we concurrently recorded the spiking activity in the dentate gyrus using GCaMP-based fiber photometry and rsfMRI data in awake rats. The correspondence between the GCaMP and global rsfMRI signals was quantified by estimating the portion of global signal peaks following

GCaMP peaks within the time window of 2-6 sec (i.e. hemodynamic delay).

To test the statistical significance of the correspondence between the calcium and global signals, we used a permutation test by randomizing the position of global signal peaks in each scan and re-calculated the co-occurrence rate between the two signals. The permutation process was repeated 1000 times to obtain the null distribution of the co-occurrence rate. This statistical test demonstrated significant correspondence between neural spikes in the hippocampus and global rsfMRI signal peaks $(p=0.016)$ (Fig. 8), again confirming that the global rsfMRI signal was associated with hippocampal neural activity.

Notably, our data showed that $\sim 35 \%$ global signal peaks were related to hippocampal spikes, but not all. To further examine this issue, we obtained the BOLD pattern of GCaMPcorresponded global signal peaks (35\% volumes, Fig. 9A) and those not corresponding to 
384 GCaMP peaks (65\% volumes, Fig. 9B). The data demonstrate that the spatial map of GCaMP385 corresponded global signal peaks was highly consistent with the global signal pattern identified

386 (Fig. 3), whereas the BOLD map of global signal peaks that did not correspond to hippocampal 387 spikes displayed a less similar pattern. It needs to be noted that the latter could still be 388 contributed by neural activity in other regions in the thalamocortical and/or hippocampal 389 networks. Indeed, some cortical activity was observed in this pattern, but the activity in the 390 hippocampus, medial prefrontal cortex and thalamus was largely absent. This pattern also 391 appears noisier, consistent with our SE-rsfMRI data which showed that $\sim 21 \%$ global signal 392 peaks were from non-neural sources (Fig. 4).

Impact of head motion and respiration

Head motion and changes of respiration rate can significantly impact the whole-brain rsfMRI data (Kalthoff et al. 2011). To rule out the potential impact of these factors on the global signal pattern, we compared the FD and respiration rate for rsfMRI frames in global signal epochs and those not in global signal epochs in ME-rsfMRI data (Fig. 10). No significant difference was observed in head motion $(p=0.61, t=0.52, \mathrm{df}=84)$ or respiration rate $(p=0.86$, $t=0.17, d f=84)$, suggesting that neither head motion nor respiration had a dominant effect on

401 the global signal pattern. In addition, the averaged motion level was on the order of $50 \mu \mathrm{m}$, far 402 less than the voxel size $\left(500 \times 500 \times 1000 \mu \mathrm{m}^{3}\right)$, suggesting that that the overall motion level 403 was low in our data.

\section{Discussion}

In the present study we systematically investigated the global brain signal in awake 
407 rodents. Using SE-rsfMRI, we found that the global signal might involve neural activity in the

408 hippocampo-cortical and thalamo-cortical networks. This finding was corroborated by ME-rsfMRI

409 data after non-neural components were extensively removed. To further validate the neural 410 origin of the global signal pattern, we simultaneously recorded calcium and rsfMRI data in awake 411 rats, and found significant correspondence between neural spikes in the hippocampus and 412 global signal peaks. Collectively, these data have filled the knowledge gap of the global brain 413 signal in awake rodents, and provided strong evidence supporting its neural basis.

Non-neural or neural?

It is well recognized that the global brain signal contains significant non-neural components including physiologic fluctuations, head motion and scanner instability (Liu et al. 2017; Nalci et al. 2017). This nature makes global signal regression an effective method to

remove artifacts in rsfMRI data. However, doubt has been casted on the validity of this preprocessing procedure as it has been shown that global signal regression mathematically

421 mandates negative correlations and might cause artifactual anticorrelated RSFC (Murphy et al. 422 2009). Studies have also shown that, besides non-neural components, the global signal has 423 neural basis (Scholvinck et al. 2010), further suggesting that global signal regression can remove 424 meaningful neural activity/connectivity, and thus bias RSFC quantification. At the functional level, 425 the global signal is tightly related to vigilance in human subjects. Wong and colleagues found 426 that the global signal amplitude was correlated to the vigilance level measured using EEG (Nalci 427 et al. 2017; Wong et al. 2012, 2013), and could be modulated using caffeine (Rack-Gomer and 428 Liu 2012). Recent studies further elucidated specific whole-brain CAPs during arousal level 429 changes that were coupled to the global signal (Liu et al. 2018; Chang et al. 2016a). Moreover, 
430 the global brain signal was found to be altered in patients with schizophrenia, suggesting that it

431 may be involved in neuropathophysiology of psychiatric disorders (Yang et al. 2017; Yang et al.

432 2014). Taken together, there is compelling evidence supporting that the global brain signal might 433 contain significant neural components in humans and anesthetized animals.

434 Consistent with this notion, our data demonstrate that the global brain signal in awake 435 rodents has important neural basis, which might involve coordinated neural activity in the 436 hippocampo-cortical and thalamo-cortical networks. First, we observed strong co-activations in 437 highly structured networks during global signal peaks in SE-rsfMRI data. These network 438 structures consistently maintained even if we clustered rsfMRI frames based on their spatial 439 similarity. Second, the same network activity was also observed in ME-rsfMRI after extensively 440 removing non-neural components. Third, the global signal peaks corresponded to 441 simultaneously measured neural spiking activity in the hippocampus. Taken together, these data 442 have provided strong evidence supporting the neural basis of the global rsfMRI signal in awake 443 rodents.

445 Functional networks involved in the global brain signal

$446 \quad$ We found two prominent functional networks co-activated during global signal peaks 447 hippocampo-cortical and thalamo-cortical networks. Strong interactions between the 448 hippocampus and the cortex have been reported in both rodent and monkey studies (Chan et al. 449 2017; Logothetis et al. 2012). Such interactions are often linked to brain-wide activity and might 450 play an important role in brain function. For instance, optogenetic stimulation of the dentate gyrus 451 was shown to enhance brain-wide functional connectivity at the resting state in rats (Chan et al. 452 2017). In monkeys, sharp-wave ripples in the hippocampus were tightly coupled to increased 
453 activity in virtually all cortical regions, which hinted the relationship of this hippocampo-cortical 454 co-activation and the global brain signal given the large portion of brain volume involved 455 (Logothetis et al. 2012; Ramirez-Villegas et al. 2015). Functionally, the activity of the 456 hippocampo-cortical network was believed to relate to the consolidation of hippocampus457 dependent memory (Ramirez-Villegas et al. 2015). Like the hippocampo-cortical network, the 458 thalamo-cortical network was also found to be involved in the global brain signal. A recent rodent 459 study reported that low-frequency optogenetic stimulations of the ventral posteromedial 460 thalamus led to brain-wide neural activity via both mono and multi-synaptic projections to cortical 461 regions, alluding the potential contribution of the thalamocortical network to the global signal 462 (Leong et al. 2016). Although specific regions involved may differ, these studies all support that 463 activities in the hippocampo-cortical and thalamo-cortical networks play a major role in the global 464 brain signal in rodents.

465 In addition to the two major functional networks, we observed remarkable involvement of 466 the basal forebrain in the global brain signal. Correspondingly, recent human (Liu et al. 2018) 467 and monkey (Turchi et al. 2018) studies demonstrated that the basal forebrain drove the brain468 wide cortical activities, especially in sensory-motor areas, and regulated the global brain signal. 469 These results collectively indicate that the global brain signal involve activities from both large470 scale neural networks and small subcortical structures.

\section{Cross-species translation}

473 There is compelling evidence demonstrating that the global brain signal is tightly related 474 to the arousal level in both humans (Wong et al. 2013; Liu et al. 2018) and monkeys (Turchi et 475 al. 2018; Chang et al. 2016a). It has been further suggested that the global brain signal and 
476 arousal were regulated by the basal forebrain (Turchi et al. 2018). Whether these results can be

477 translated to rodents is an interesting topic to investigate and certainly warrants more detailed

478 studies in the future. However, some differences between these species that might affect the 479 translation of the global brain signal need to be acknowledged. In particular, in rodents two thirds 480 of the brain volume are subcortical regions and one third is the cortex. This composition is in 481 remarkable contrast to primates/humans, in which cortical regions occupy the vast majority of 482 the brain volume. Given that the global brain signal is defined by the averaged activity across 483 the whole brain, it would have very different contributions from the cortex versus subcortex 484 between rodents and primates/humans. Therefore, it should not be assumed that our findings in 485 the global brain signal can be directly translated to other species.

\section{Potential pitfalls and limitations}

There are a number of physiological factors that can affect rsfMRI signal and can potentially impact the global rsfMRI pattern, such as respiratory volume variability, vasomotion, 490 brain pulsation, blood pressure, just to name a few. Although our study has been carefully 491 designed to rule out these possibilities, including using stringent motion control, regressing out 492 signals from the white matter and ventricles, using ME-EPI to separate non-neural components 493 and simultaneously recording GCaMP signal in the hippocampus, caution still needs to be taken 494 for the interpretation of the results. Specifically, it is difficult to conclude that the global signal 495 pattern revealed is completely free from non-neural artifacts. For instance, some of the 496 physiological factors are directly related to vascular changes, which also contribute to T2* 497 changes, and thus cannot be ruled out by ME-EPI. In addition, signals from large veins can 498 contribute to the global rsfMRI signal. Nonetheless, given that the consistency of multiple 
499 measurements (i.e. SE-EPI, ME-EPI and GCaMP signal), it is unlikely physiologic artifacts 500 dominated the global brain signal pattern.

Although ME-rsfMRI has the advantage of differentiating neural and non-neural 502 components, this method cannot guarantee each ICA component was completely neural or non-

503 neural. In fact, all ICA components showed non-zero $\kappa$ and $\rho$ values, suggesting that they were 504 more or less mixed with neural and non-neural constituents. In this study, we used stringent 505 criteria to select neural components. Therefore, it is likely that some non-neural ICA components 506 identified contained some neural elements. Nonetheless, the purpose of utilizing ME-EPI in this 507 study is to extensively regress out non-neural components, in order to validate the results found 508 in SE-rsfMRI data. Indeed, 28 regressors were used for de-noising ME-rsfMRI data, compared 509 to only seven regressors used in preprocessing SE-rsfMRI data. Even with such stringent criteria, 510 consistent results were obtained between SE-rsfMRI and ME-rsfMRI data, confirming that 511 activities in the hippocampo-cortical and thalamo-cortical networks indeed represent neural 512 contributions to the global signal in awake rats.

513 The present study focused on revealing the spatial pattern of brain activity when global 514 signal was high. An equally important issue is to understand the temporal dynamics of the global 515 signal, and a number of advanced analysis method has been applied. For instance, a recent 516 study investigated the relationship between separate co-activation patterns and the phase of 517 global signal (Gutierrez-Barragan et al. 2019). This issue needs to be specifically studied in the 518 future.

\section{Conclusion}


suggest that the hippocampo-cortical and thalamo-cortical networks play a major role in the neural basis of the global signal. These results have filled our knowledge gap of the global brain signal in awake rodents and provided important insight into its neural substrate. The present study can further facilitate comparative studies investigating the generalized function the global signal may have across rodents and humans.

\section{References:}

Aguirre GK, Zarahn E, D'Esposito M (1997) Empirical analyses of BOLD fMRI statistics. II. Spatially smoothed data collected under null-hypothesis and experimental conditions. Neuroimage 5 (3):199-212

Bergmann E, Zur G, Bershadsky G, Kahn I (2016) The Organization of Mouse and Human Cortico-Hippocampal Networks Estimated by Intrinsic Functional Connectivity. Cereb Cortex 26 (12):4497-4512. doi:10.1093/cercor/bhw327

Birn RM, Smith MA, Jones TB, Bandettini PA (2008) The respiration response function: the temporal dynamics of $\mathrm{fMRI}$ signal fluctuations related to changes in respiration. Neuroimage 40 (2):644-654. doi:10.1016/j.neuroimage.2007.11.059

Biswal B, Yetkin FZ, Haughton VM, Hyde JS (1995) Functional connectivity in the motor cortex of resting human brain using echo-planar MRI. Magn Reson Med 34 (4):537-541

Biswal BB, Mennes M, Zuo XN, Gohel S, Kelly C, Smith SM, Beckmann CF, Adelstein JS, Buckner RL, Colcombe S, Dogonowski AM, Ernst M, Fair D, Hampson M, Hoptman MJ, Hyde JS, Kiviniemi VJ, Kotter R, Li SJ, Lin CP, Lowe MJ, Mackay C, Madden DJ, Madsen KH, Margulies DS, Mayberg HS, McMahon K, Monk CS, Mostofsky SH, Nagel BJ, Pekar JJ, Peltier SJ, Petersen SE, Riedl V, Rombouts SA, Rypma B, Schlaggar BL, Schmidt S, Seidler RD, Siegle GJ, Sorg C, Teng GJ, Veijola J, Villringer A, Walter M, Wang L, Weng XC, Whitfield-Gabrieli S, Williamson P, Windischberger C, Zang YF, Zhang HY, Castellanos FX, Milham MP (2010) Toward discovery science of human brain function. Proc Natl Acad Sci U S A 107 (10):4734-4739. doi:10.1073/pnas.0911855107

Calhoun VD, Adali T, Pearlson GD, Pekar JJ (2001) A method for making group inferences from functional MRI data using independent component analysis. Hum Brain Mapp 14 (3):140151 
Chan RW, Leong ATL, Ho LC, Gao PP, Wong EC, Dong CM, Wang X, He J, Chan YS, Lim LW, Wu EX (2017) Low-frequency hippocampal-cortical activity drives brain-wide resting-state functional MRI connectivity. Proc Natl Acad Sci U S A 114 (33):E6972-E6981. doi:10.1073/pnas.1703309114

Chang C, Cunningham JP, Glover GH (2009) Influence of heart rate on the BOLD signal: the $\begin{array}{lllll}\text { cardiac } & \text { response } & \text { (3):857-869. }\end{array}$ doi:10.1016/j.neuroimage.2008.09.029

Chang C, Leopold DA, Scholvinck ML, Mandelkow H, Picchioni D, Liu X, Ye FQ, Turchi JN, Duyn $\mathrm{JH}$ (2016a) Tracking brain arousal fluctuations with fMRI. Proc Natl Acad Sci U S A 113 (16):4518-4523. doi:10.1073/pnas.1520613113

Chang PC, Procissi D, Bao Q, Centeno MV, Baria A, Apkarian AV (2016b) Novel method for functional brain imaging in awake minimally restrained rats. J Neurophysiol 116 (1):6180. doi:10.1152/jn.01078.2015

Ciric R, Rosen AFG, Erus G, Cieslak M, Adebimpe A, Cook PA, Bassett DS, Davatzikos C, Wolf $\mathrm{DH}$, Satterthwaite TD (2018) Mitigating head motion artifact in functional connectivity MRI. Nat Protoc 13 (12):2801-2826. doi:10.1038/s41596-018-0065-y

Dopfel D, Perez PD, Verbitsky A, Bravo-Rivera H, Ma Y, Quirk GJ, Zhang N (2019) Individual variability in behavior and functional networks predicts vulnerability using an animal model of PTSD. Nat Commun 10 (1):2372. doi:10.1038/s41467-019-09926-z

Dopfel D, Zhang N (2018) Mapping stress networks using functional magnetic resonance imaging in awake animals. Neurobiol Stress 9:251-263. doi:10.1016/j.ynstr.2018.06.002

Fox MD, Snyder AZ, Vincent JL, Corbetta M, Van Essen DC, Raichle ME (2005) The human brain is intrinsically organized into dynamic, anticorrelated functional networks. Proc Natl Acad Sci U S A 102 (27):9673-9678

Gao YR, Ma Y, Zhang Q, Winder AT, Liang Z, Antinori L, Drew PJ, Zhang N (2016) Time to wake up: Studying neurovascular coupling and brain-wide circuit function in the unanesthetized animal. Neuroimage. doi:10.1016/j.neuroimage.2016.11.069

Gutierrez-Barragan D, Basson MA, Panzeri S, Gozzi A (2019) Infraslow State Fluctuations Govern Spontaneous fMRI Network Dynamics. Curr Biol. doi:10.1016/j.cub.2019.06.017 Hamilton C, Ma Y, Zhang N (2017) Global reduction of information exchange during anestheticinduced unconsciousness. Brain structure \& function 222 (7):3205-3216. doi:10.1007/s00429-017-1396-0

Kalthoff D, Seehafer JU, Po C, Wiedermann D, Hoehn M (2011) Functional connectivity in the rat at 11.7T: Impact of physiological noise in resting state fMRI. Neuroimage 54 (4):28282839. doi:S1053-8119(10)01345-5 [pii]10.1016/j.neuroimage.2010.10.053

Kim CK, Yang SJ, Pichamoorthy N, Young NP, Kauvar I, Jennings JH, Lerner TN, Berndt A, Lee SY, Ramakrishnan C, Davidson TJ, Inoue M, Bito H, Deisseroth K (2016) Simultaneous fast measurement of circuit dynamics at multiple sites across the mammalian brain. Nature methods 13 (4):325-328. doi:10.1038/nmeth.3770 
Kundu P, Brenowitz ND, Voon V, Worbe Y, Vertes PE, Inati SJ, Saad ZS, Bandettini PA, Bullmore ET (2013) Integrated strategy for improving functional connectivity mapping using multiecho fMRI. Proc Natl Acad Sci U S A 110 (40):16187-16192. doi:10.1073/pnas.1301725110

Kundu P, Inati SJ, Evans JW, Luh WM, Bandettini PA (2012) Differentiating BOLD and nonBOLD signals in fMRI time series using multi-echo EPI. Neuroimage 60 (3):1759-1770. doi:10.1016/j.neuroimage.2011.12.028

Kundu P, Santin MD, Bandettini PA, Bullmore ET, Petiet A (2014) Differentiating BOLD and nonBOLD signals in fMRI time series from anesthetized rats using multi-echo $\mathrm{EPI}$ at $11.7 \mathrm{~T}$. Neuroimage 102 Pt 2:861-874. doi:10.1016/j.neuroimage.2014.07.025

Leong AT, Chan RW, Gao PP, Chan YS, Tsia KK, Yung WH, Wu EX (2016) Long-range projections coordinate distributed brain-wide neural activity with a specific spatiotemporal profile. Proc Natl Acad Sci U S A 113 (51):E8306-E8315. doi:10.1073/pnas.1616361113

Liang Z, King J, Zhang N (2011) Uncovering intrinsic connectional architecture of functional networks in awake rat brain. J Neurosci 31 (10):3776-3783

Liang Z, King J, Zhang N (2012a) Anticorrelated resting-state functional connectivity in awake rat brain. Neuroimage 59 (2):1190-1199. doi:S1053-8119(11)00896-2 [pii]10.1016/j.neuroimage.2011.08.009

Liang Z, King J, Zhang N (2012b) Intrinsic organization of the anesthetized brain. J Neurosci 32 (30):10183-10191. doi:10.1523/JNEUROSCI.1020-12.2012

Liang Z, King J, Zhang N (2014) Neuroplasticity to a single-episode traumatic stress revealed by resting-state $\mathrm{fMRI}$ in awake rats. Neuroimage 103:485-491. doi:10.1016/j.neuroimage.2014.08.050

Liang Z, Li T, King J, Zhang N (2013) Mapping thalamocortical networks in rat brain using resting-state functional connectivity. Neuroimage 83:237-244. doi:10.1016/j.neuroimage.2013.06.029

Liang Z, Liu X, Zhang N (2015a) Dynamic resting state functional connectivity in awake and anesthetized rodents. Neuroimage 104:89-99. doi:10.1016/j.neuroimage.2014.10.013

Liang Z, Ma Y, Watson GDR, Zhang N (2017) Simultaneous GCaMP6-based fiber photometry and fMRI in rats. J Neurosci Methods 289:31-38. doi:10.1016/j.jneumeth.2017.07.002

Liang Z, Watson GD, Alloway KD, Lee G, Neuberger T, Zhang N (2015b) Mapping the functional network of medial prefrontal cortex by combining optogenetics and fMRI in awake rats. Neuroimage 117:114-123. doi:10.1016/j.neuroimage.2015.05.036

Liu TT (2016) Noise contributions to the fMRI signal: An overview. Neuroimage 143:141-151. doi:10.1016/j.neuroimage.2016.09.008

Liu TT, Nalci A, Falahpour M (2017) The global signal in fMRI: Nuisance or Information? Neuroimage 150:213-229. doi:10.1016/j.neuroimage.2017.02.036

Liu X, de Zwart JA, Scholvinck ML, Chang C, Ye FQ, Leopold DA, Duyn JH (2018) Subcortical evidence for a contribution of arousal to fMRI studies of brain activity. Nat Commun 9 (1):395. doi:10.1038/s41467-017-02815-3 
633 Liu Y, Zhang N (2019) Propagations of spontaneous brain activity in awake rats. Neuroimage 202:116176. doi:10.1016/j.neuroimage.2019.116176

Logothetis NK, Eschenko O, Murayama Y, Augath M, Steudel T, Evrard HC, Besserve M, Oeltermann A (2012) Hippocampal-cortical interaction during periods of subcortical silence. Nature 491 (7425):547-553. doi:10.1038/nature11618

Ma Y, Hamilton C, Zhang N (2017) Dynamic Connectivity Patterns in Conscious and Unconscious Brain. Brain connectivity 7 (1):1-12. doi:10.1089/brain.2016.0464

Ma Y, Shaik MA, Kozberg MG, Kim SH, Portes JP, Timerman D, Hillman EM (2016) Restingstate hemodynamics are spatiotemporally coupled to synchronized and symmetric neural activity in excitatory neurons. Proc Natl Acad Sci U S A 113 (52):E8463-E8471. doi:10.1073/pnas.1525369113

Ma Z, Perez P, Ma Z, Liu Y, Hamilton C, Liang Z, Zhang N (2018) Functional atlas of the awake rat brain: A neuroimaging study of rat brain specialization and integration. Neuroimage 170:95-112. doi:10.1016/j.neuroimage.2016.07.007

Ma Z, Zhang N (2018) Temporal transitions of spontaneous brain activity. eLife 7. doi:10.7554/eLife.33562

Matsui T, Murakami T, Ohki K (2016) Transient neuronal coactivations embedded in globally propagating waves underlie resting-state functional connectivity. Proc Natl Acad Sci U S A 113 (23):6556-6561. doi:10.1073/pnas.1521299113

Murphy K, Birn RM, Handwerker DA, Jones TB, Bandettini PA (2009) The impact of global signal regression on resting state correlations: are anti-correlated networks introduced? Neuroimage 44 (3):893-905

Nalci A, Rao BD, Liu TT (2017) Global signal regression acts as a temporal downweighting process in resting-state fMRI. Neuroimage 152:602-618. doi:10.1016/j.neuroimage.2017.01.015

Perez PD, Ma Z, Hamilton C, Sanchez C, Mork A, Pehrson AL, Bundgaard C, Zhang N (2018) Acute effects of vortioxetine and duloxetine on resting-state functional connectivity in the awake rat. Neuropharmacology 128:379-387. doi:10.1016/j.neuropharm.2017.10.038

Power JD, Barnes KA, Snyder AZ, Schlaggar BL, Petersen SE (2012) Spurious but systematic correlations in functional connectivity MRI networks arise from subject motion. Neuroimage 59 (3):2142-2154. doi:10.1016/j.neuroimage.2011.10.018

Power JD, Schlaggar BL, Petersen SE (2015) Recent progress and outstanding issues in motion correction in resting state fMRI. Neuroimage 105:536-551. doi:10.1016/j.neuroimage.2014.10.044

Rack-Gomer AL, Liu TT (2012) Caffeine increases the temporal variability of resting-state BOLD connectivity in the motor cortex. Neuroimage 59 (3):2994-3002. doi:10.1016/j.neuroimage.2011.10.001

Raichle ME (2006) Neuroscience. The brain's dark energy. Science 314 (5803):1249-1250. doi:10.1126/science. 1134405

Raichle ME (2010) The brain's dark energy. Sci Am 302 (3):44-49 
673

674

675

676

677

678

679

680

681

682

683

684

685

686

687

688

689

690

691

692

693

694

695

696

697

698

699

700

701

702

703

704

705

706

707

708

709

710

711

Ramirez-Villegas JF, Logothetis NK, Besserve M (2015) Diversity of sharp-wave-ripple LFP signatures reveals differentiated brain-wide dynamical events. Proc Natl Acad Sci U S A 112 (46):E6379-6387. doi:10.1073/pnas.1518257112

Rivera B, Miller S, Brown E, Price R (2005) A novel method for endotracheal intubation of mice and rats used in imaging studies. Contemp Top Lab Anim Sci 44 (2):52-55

Satterthwaite TD, Wolf DH, Loughead J, Ruparel K, Elliott MA, Hakonarson H, Gur RC, Gur RE (2012) Impact of in-scanner head motion on multiple measures of functional connectivity: relevance for studies of neurodevelopment in youth. Neuroimage 60 (1):623-632. doi:10.1016/j.neuroimage.2011.12.063

Scholvinck ML, Maier A, Ye FQ, Duyn JH, Leopold DA (2010) Neural basis of global restingstate fMRI activity. Proc Natl Acad Sci U S A 107 (22):10238-10243

Smith JB, Liang Z, Watson GDR, Alloway KD, Zhang N (2017) Interhemispheric resting-state functional connectivity of the claustrum in the awake and anesthetized states. Brain structure \& function 222 (5):2041-2058. doi:10.1007/s00429-016-1323-9

Smith SM, Fox PT, Miller KL, Glahn DC, Fox PM, Mackay CE, Filippini N, Watkins KE, Toro R, Laird AR, Beckmann CF (2009) Correspondence of the brain's functional architecture during activation and rest. Proc Natl Acad Sci U S A 106 (31):13040-13045. doi:10.1073/pnas.0905267106

0905267106 [pii]

Turchi J, Chang C, Ye FQ, Russ BE, Yu DK, Cortes CR, Monosov IE, Duyn JH, Leopold DA (2018) The Basal Forebrain Regulates Global Resting-State fMRI Fluctuations. Neuron 97 (4):940-952 e944. doi:10.1016/j.neuron.2018.01.032

Van Dijk KR, Sabuncu MR, Buckner RL (2012) The influence of head motion on intrinsic functional connectivity MRI. doi:10.1016/j.neuroimage.2011.07.044

Wen H, Liu Z (2016) Broadband Electrophysiological Dynamics Contribute to Global RestingState fMRI Signal. J Neurosci 36 (22):6030-6040. doi:10.1523/JNEUROSCI.018716.2016

Wong CW, Olafsson V, Tal O, Liu TT (2012) Anti-correlated networks, global signal regression, and the effects of caffeine in resting-state functional MRI. Neuroimage 63 (1):356-364. doi:10.1016/j.neuroimage.2012.06.035

Wong CW, Olafsson V, Tal O, Liu TT (2013) The amplitude of the resting-state fMRI global signal is related to EEG vigilance measures. Neuroimage 83:983-990. doi:10.1016/j.neuroimage.2013.07.057

Yang GJ, Murray JD, Glasser M, Pearlson GD, Krystal JH, Schleifer C, Repovs G, Anticevic A (2017) Altered Global Signal Topography in Schizophrenia. Cereb Cortex 27 (11):51565169. doi:10.1093/cercor/bhw297

Yang GJ, Murray JD, Repovs G, Cole MW, Savic A, Glasser MF, Pittenger C, Krystal JH, Wang XJ, Pearlson GD, Glahn DC, Anticevic A (2014) Altered global brain signal in 
$\begin{array}{llllllll}\text { schizophrenia. Proc Natl Acad Sci U S A } 111 & \text { (20):7438-7443. }\end{array}$ doi:10.1073/pnas.1405289111

Yoshida K, Mimura Y, Ishihara R, Nishida H, Komaki Y, Minakuchi T, Tsurugizawa T, Mimura M, Okano H, Tanaka KF, Takata N (2016) Physiological effects of a habituation procedure for functional MRI in awake mice using a cryogenic radiofrequency probe. J Neurosci Methods 274:38-48. doi:10.1016/j.jneumeth.2016.09.013

Yushkevich PA, Piven J, Hazlett HC, Smith RG, Ho S, Gee JC, Gerig G (2006) User-guided 3D active contour segmentation of anatomical structures: significantly improved efficiency and reliability. Neuroimage 31 (3):1116-1128. doi:10.1016/j.neuroimage.2006.01.015 
A

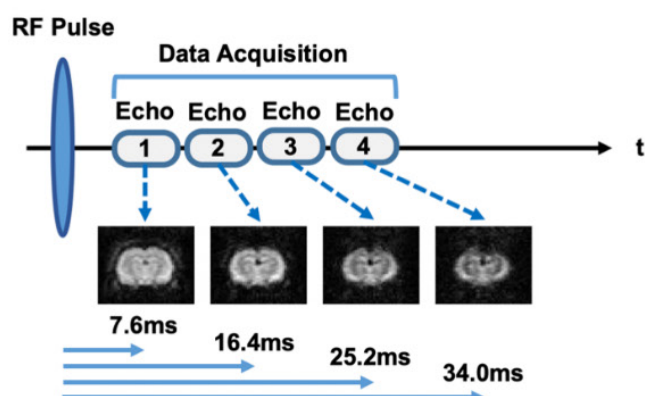

C

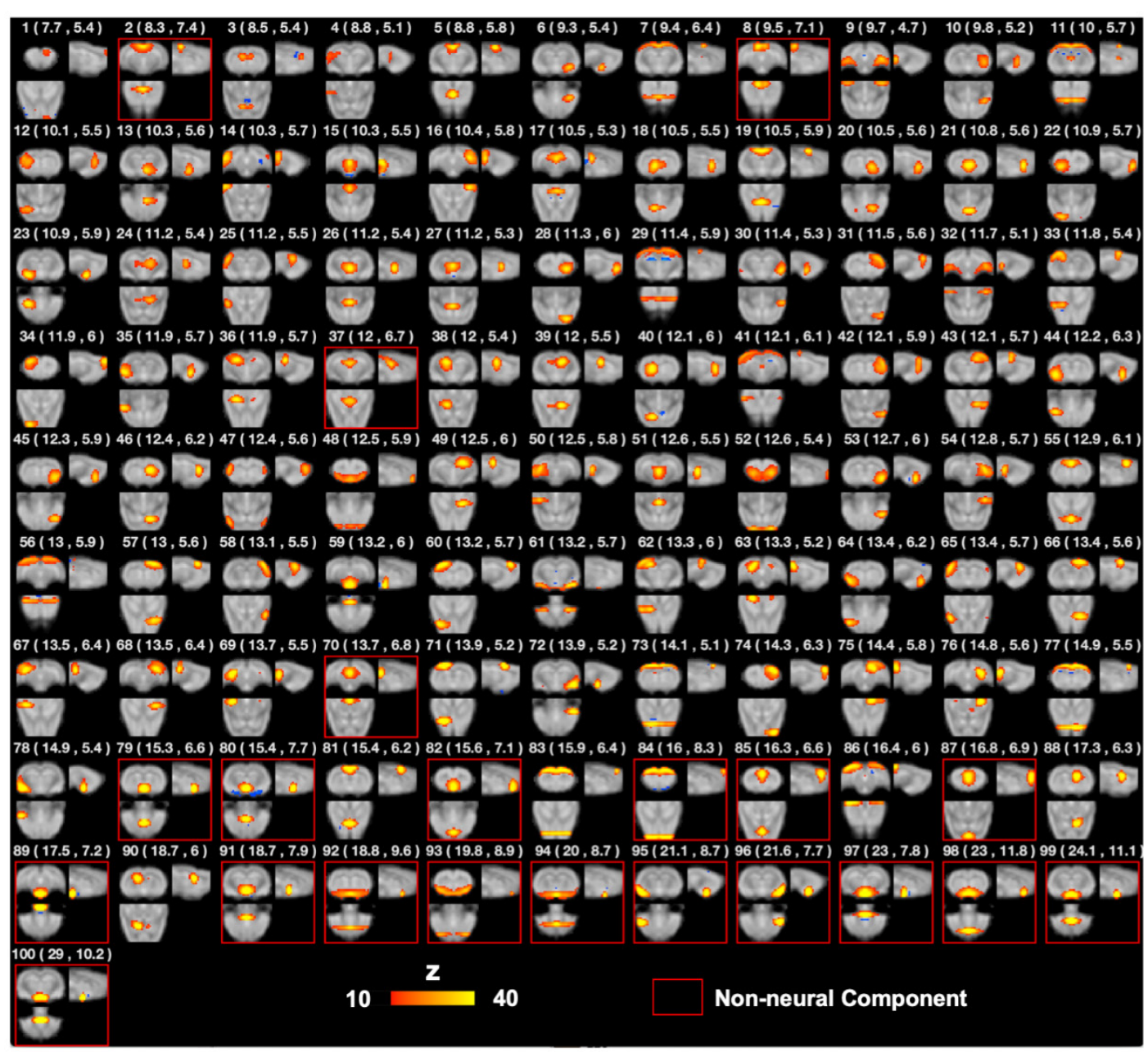

B

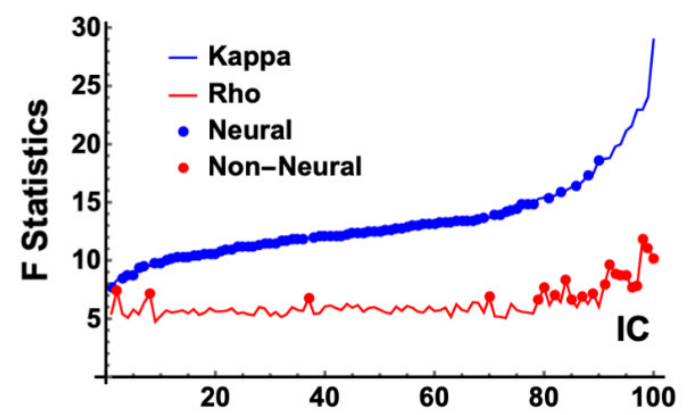


A

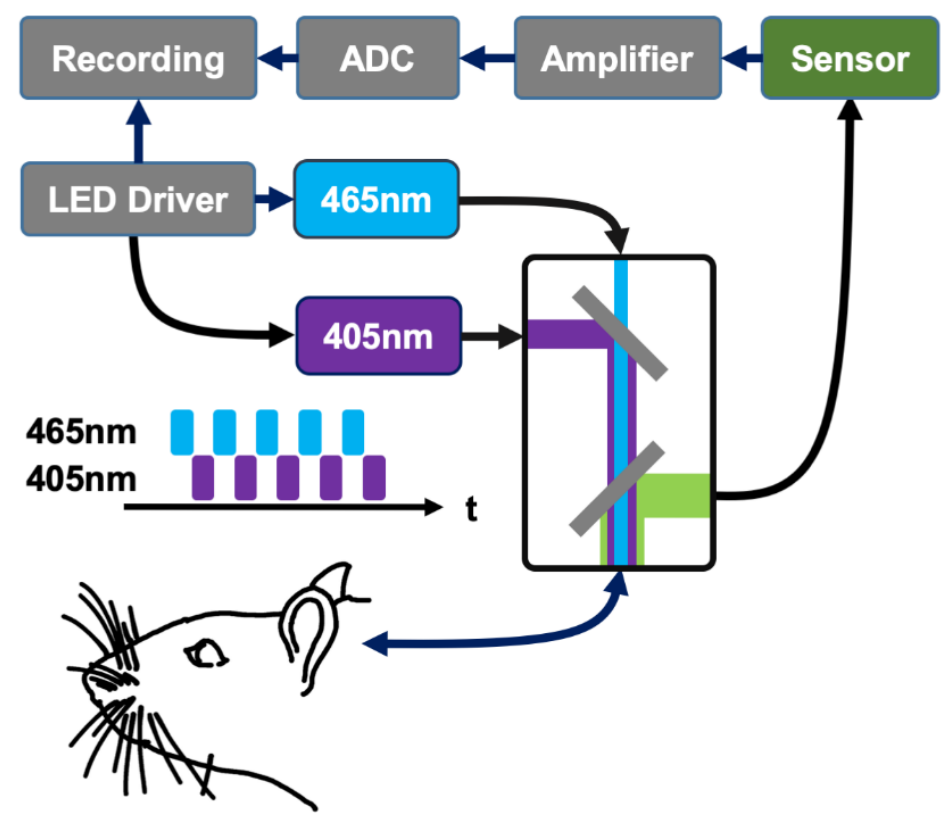

B
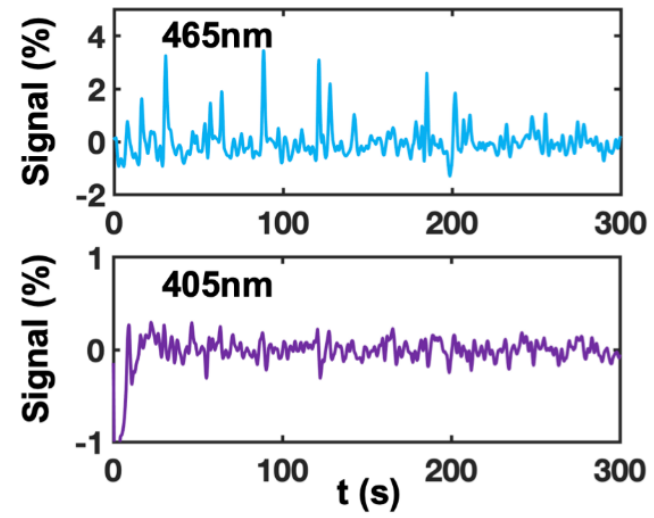

C

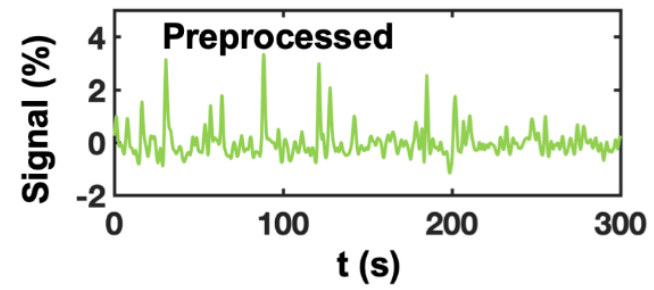

Figure 2. Setup of GCaMP fiber photometry and signal preprocessing. (A) Setup of the twowavelength GCaMP fiber photometry system. (B) Signals detected with $465 \mathrm{~nm}$ and 405 $\mathrm{nm}$ excitation wavelengths, respectively. (C) $405 \mathrm{~nm}$ signals were regressed out from 465 $\mathrm{nm}$ signals to provide fluorescence changes pertinent to neuronal activity. 


\section{A}

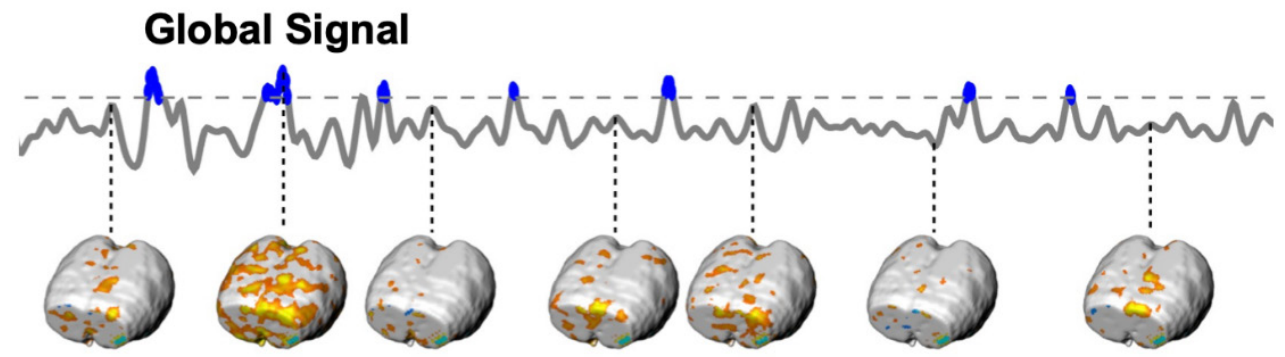

B

C
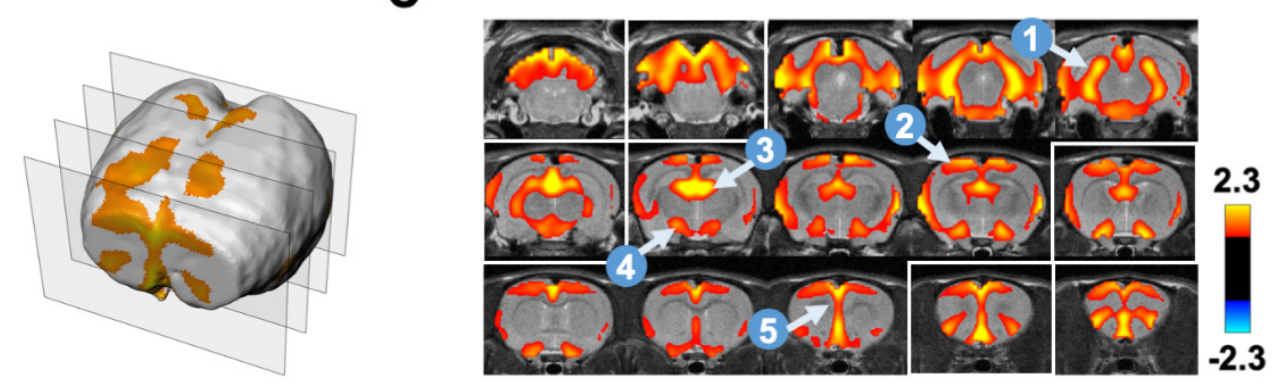

Figure 3. Averaged co-activation pattern (CAP) during global signal peaks in SE-rsfMRI data. (A) Extraction of rsfMRI volumes during global signal peaks. (B) 3D visualization of the averaged CAP during global signal peaks. Positions of the four slices selected were highlighted in white boxes in (C). (C) Slice-by-slice view of the global signal CAP. Five brain regions highlighted include: 1 , hippocampus; 2 , sensory-motor cortex; 3 , medial dorsal thalamus; 4 , basal forebrain; 5 , prefrontal cortex. 
A
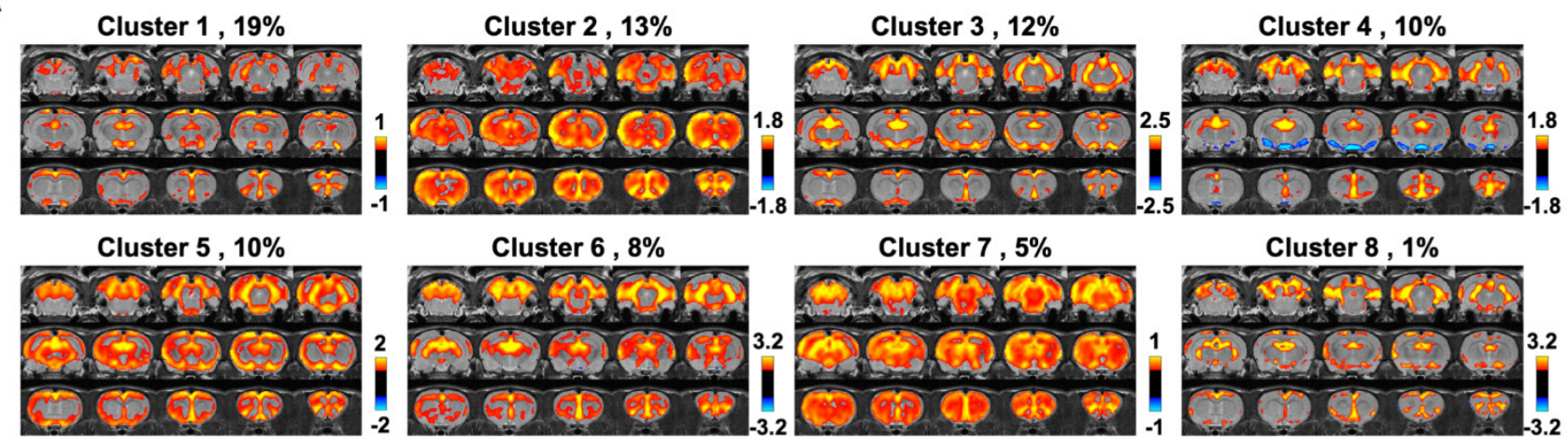

B
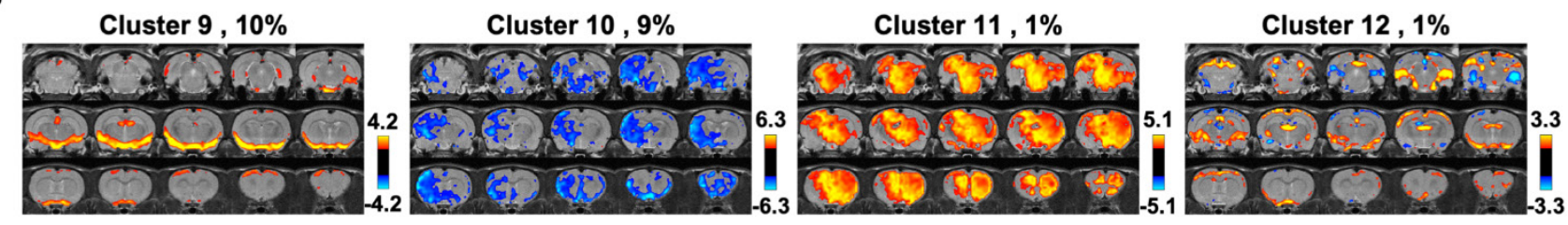

Figure 4. Clustering rsfMRI frames during global signal peaks. (A) Clusters resembling the mean global signal CAP. (B) Clusters exhibiting distinct CAPs. The occurrence rate is displayed next to the cluster number. 


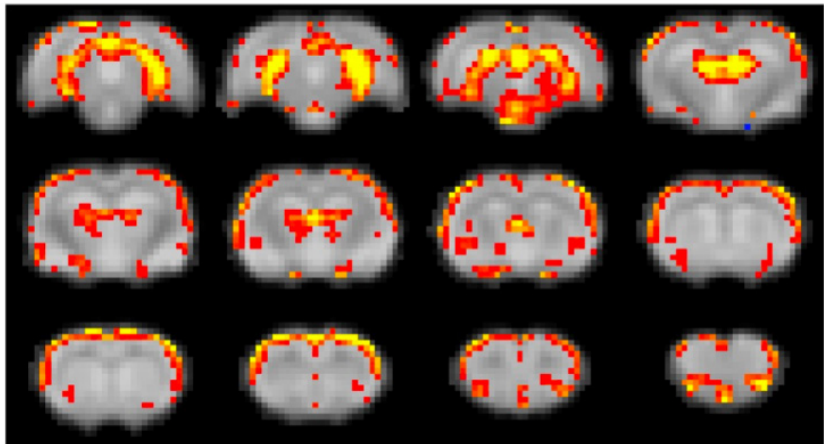

0.8

Figure 5. Co-activation pattern during global signal peaks in de-noised ME-rsfMRI data. 
A $\quad$ B

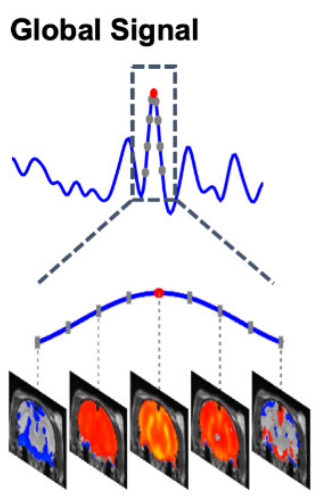

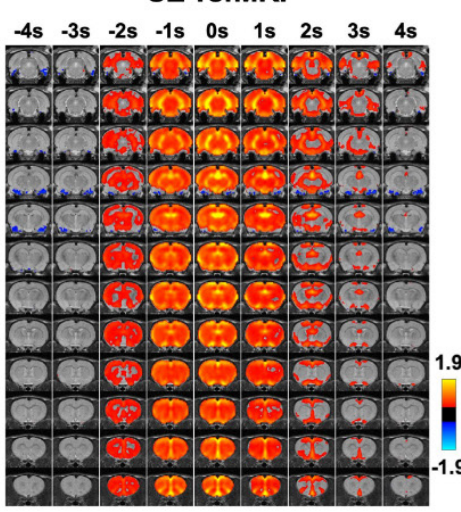

C

\section{ME-rsfMRI}

$-6 \mathrm{~s} \quad-4.5 \mathrm{~s}-3 \mathrm{~s} \quad-1.5 \mathrm{~s} \quad 0 \mathrm{~s} \quad 1.5 \mathrm{~s} \quad 3 \mathrm{~s} \quad 4.5 \mathrm{~s} \quad 6 \mathrm{~s}$
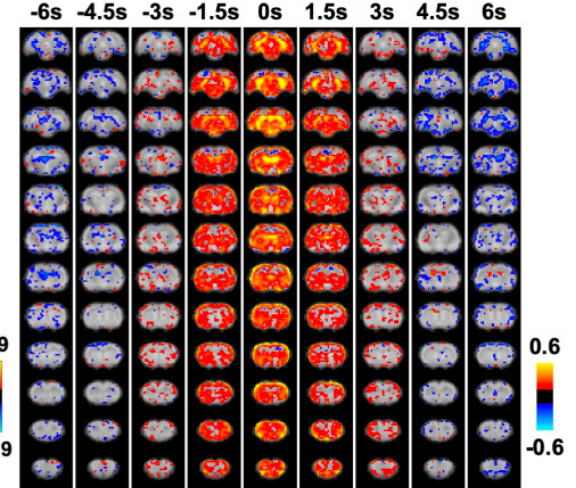

D

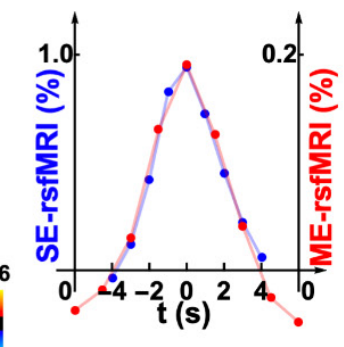

Figure 6. Spatiotemporal dynamics of the global signal. (A) An example of a global signal epoch including $8 \mathrm{rsfMRI}$ volumes (gray dots) surrounding a local peak (red dot). (B) Averaged spatiotemporal pattern of global signal epochs in SE-rsfMRI data. Each column represents the averaged spatial pattern of global signal epochs at a time point. (C) Averaged spatiotemporal pattern of global signal epochs in ME-rsfMRI data. (D) Frameby-frame global signal amplitude averaged across all epochs in SE and ME-rsfMRI data, respectively. 
A

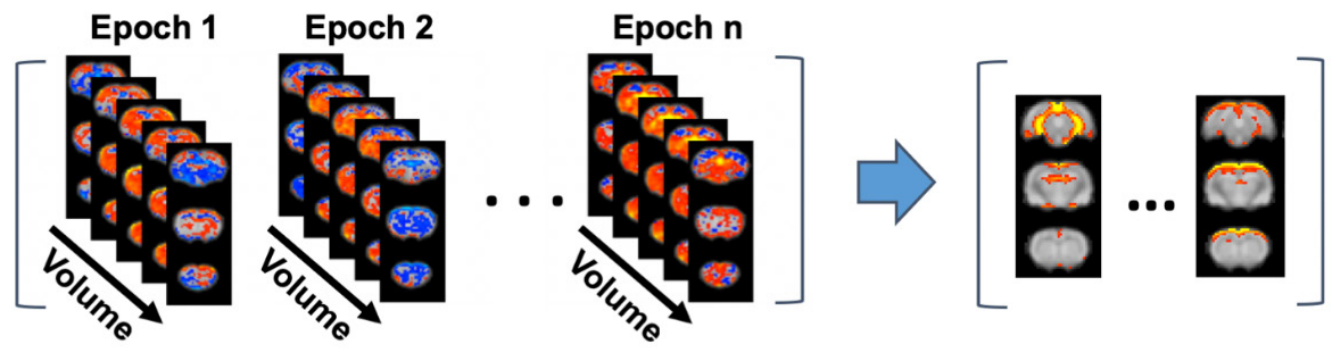

B

\section{IC 1}

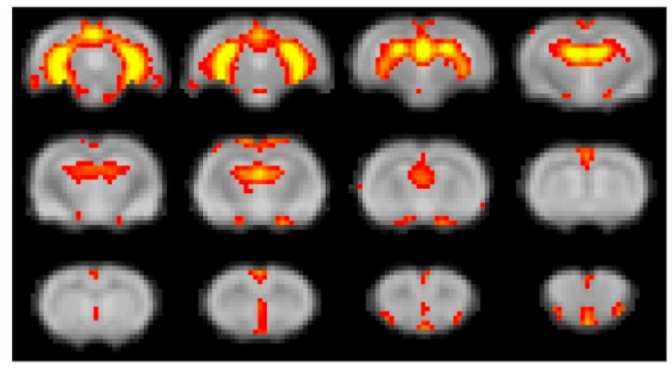

IC 2

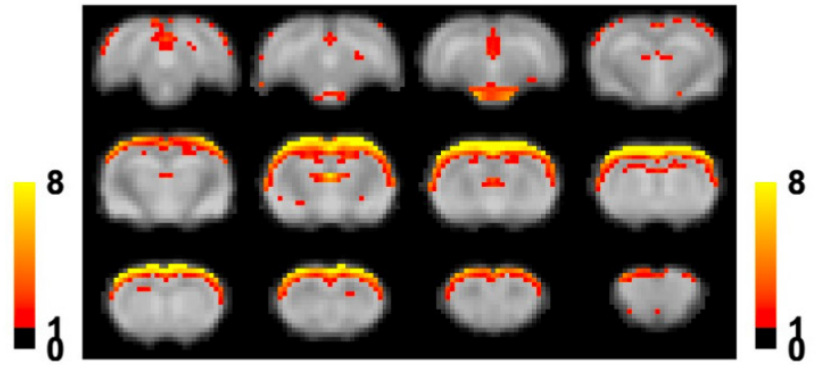

Figure 7 Major functional networks involved in the global signal. (A) Spatial group-ICA applied to all global signal epochs obtained using ME-rsfMRI data. (B) Two major functional networks derived from group ICA. 
A

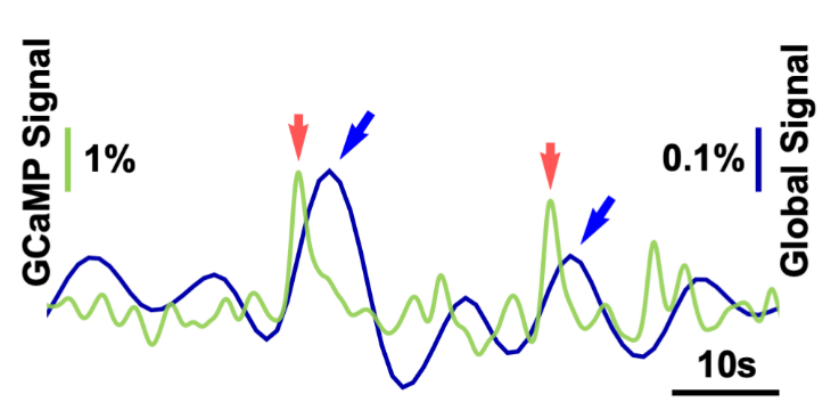

B

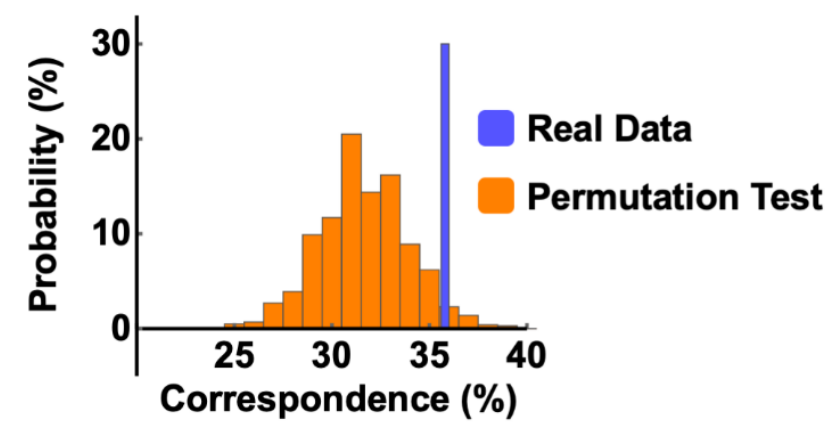

773

774

775

776

777

778

779
Figure 8. Correspondence between the global rsfMRI signal and neuronal spikes in the hippocampus. (A) Representative time courses of the global rsfMRI signal (blue) and calcium signal (green). Global signal peaks (blue arrows) and neural spikes (red arrows) in the hippocampus were strongly coupled within $2-6 \mathrm{sec}$ of hemodynamic delay. (B) Permutation test of the correspondence between the global signal and GCaMP peaks ( $p$ $=0.016,1000$ permutations). 


\section{A}

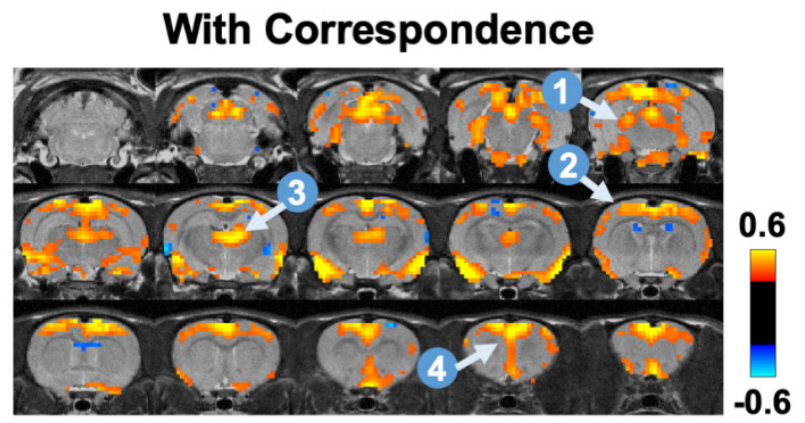

B

Without Correspondence

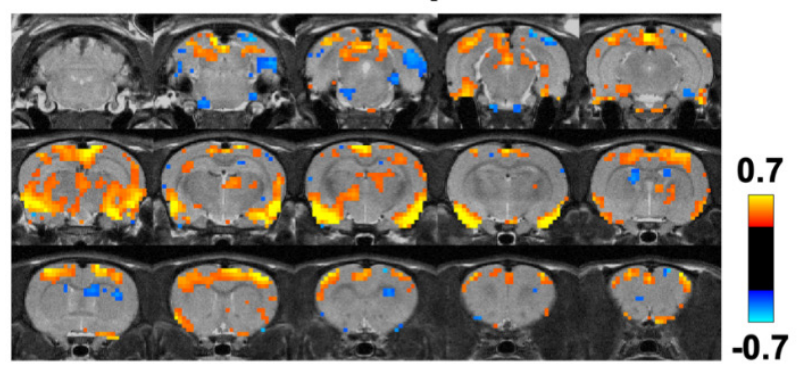

Figure 9. BOLD co-activation patterns during global signal peaks with (A) and without (B) corresponding GCaMP peaks in the hippocampus. Four brain regions highlighted include: 1 , hippocampus; 2 , sensory-motor cortex; 3 , medial dorsal thalamus; 4 , prefrontal cortex. 

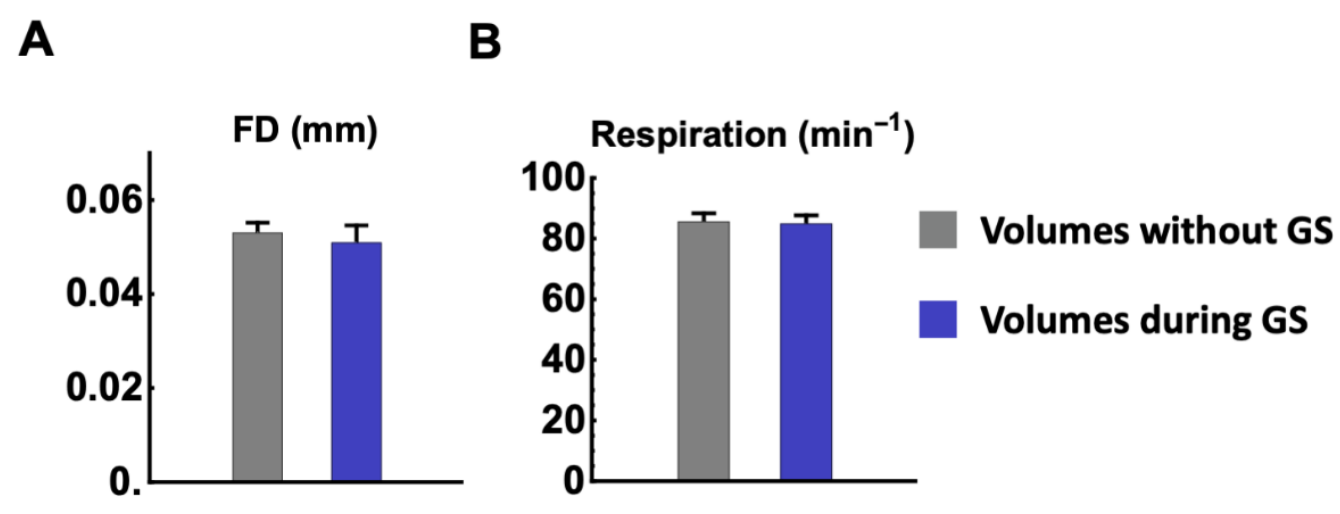

Figure 10. Effects of head motion and respiration. (A) Framewise displacement (FD) and (B) respiration rate for rsfMRI frames in global signal epochs (blue bars) and rsfMRI frames not in global signal epochs (gray bars), respectively. No significant difference was found 790 in head motion level $(p=0.61)$ or respiration $(p=0.86)$. 